\title{
Metric of global texture direction Based on wavelet modulus maxima threshold selection and Power Spectrum
}

\author{
Chen $\mathrm{Ye}^{1, \mathrm{a}}$, Jin peng ${ }^{2, \mathrm{~b}}$ and Yuan Xiaoping ${ }^{3, \mathrm{c}}$ \\ ${ }^{1}$ China University of Mining and Technology, Xuzhou 221008, China \\ ${ }^{2}$ China University of Mining and Technology, Xuzhou 221008, China \\ ${ }^{3}$ China University of Mining and Technology, Xuzhou 221008, China \\ a cumtchenye@163.com, ${ }^{b}$ jinpeng_kd@163.com, ${ }^{\circ}$ xpyuankd@163.com
}

\begin{abstract}
Keywords: Texture orientation, Gabor Filter, Global texture direction, Feature extraction Abstract. The present study proposes a metric algorithm for image texture orientation to establish the corresponding mathematical model. First, the image power spectrum is obtained through wavelet maximum edge detection and edge expansion of the texture image. Then threshold image power is set to acquire multiple sets of texture power vector. Then the sum of texture power vector sets makes global power vector, quantitative value of global texture direction. At last, precision of sector and global texture direction under different threshold values is tested on multiple linear stripe and wood texture images. Test results on these two texture databases have a high consistency with human visual perception. It can be concluded that the proposed metric algorithm of texture direction measurement can satisfy the need of high accuracy of man-machine texture recognition model.
\end{abstract}

\section{Introduction}

As a description to distinguish significant characteristics of object and area, an important image information consist of the texture. Texture research purpose is to extract images, described the essential characteristics of the object, and then segmenting image, classification and recognition that describing the object's mathematical characteristics. The research of the main direction of texture can calculate the goal displacement on image, restoring image via the main texture direction, forecasting the weather by the satellite cloud, calculating the direction and intensity of erosion phenomenon and the relative displacement between the subject and target on image. It also calculates surface contour and wood processing according to the texture direction. Therefore, the research of the main direction of texture has broad application prospects.

Research shows that periodic, orientation and randomness are the most important three factors to representation of texture. There are many literatures of the direction of the texture and the expression method. It has been proposed based on 2Dwold transform texture model. The texture is decomposed for periodic components, directional composition and random component in the model. Testing texture direction based on Tamura, the calculation is simple, but the direction measure precision not enough, especially for arrangement irregular texture image is not sensitive. Using parallelogram expression texture directivity is proposed in [4]. Two adjacent sides of parallelogram is direction of the texture composition. Based on difference function, [5] shows the method of calculation an image texture directional size according to the research for an image's texture by Karu. In addition, [6] put forward a method to extract texture area directional component according to texture of second order or higher order statistics characteristic.

To sum up, most of the texture directional measure method in essence are based on the classical statistics, focusing on the texture local mode and the description of the rules, and did not reflect the texture as a global pattern directional representation. And this kind of directional representation is consistent with human visual perception of the directional texture. The emphasis of the paper tired to give a global texture directional measurement. This paper focus on the wavelet maxima edge detection with multi-scale and multidirectional measurement features, combined with the power spectrum, obtained a description of the global grain orientation power vector and measure algorithm. 
And with Brodatz grain image source as experimental material gives the measurement model of the global texture direction.

\section{Edge detection}

The edge is the most important characteristics of the wood texture to outline the geometric characteristics of the texture, pass the trees to grow a variety of information, and is an important feature of the description of the wood texture direction. It provides valuable and important characteristic parameter for the analysis of the main texture of wood.

The traditional texture detection achieved method is by means of the airspace differential operator for convolution, played the role of the high pass filter. The operator usually use near the edge of the first or second order derivative changing characteristics, so is often sensitive to variation of image noise. Not only is the efficiency of noise reduction poor, and easy to import noise. And the edge of the wavelet analysis has many advantages of resolution and reduces the noise.

Because every texture separated from another and their points often represent every texture direction, so this paper adopted multiscale modulus maxima of wavelet transform edge detection method.

\section{Algorithm Principle}

Let as a two-dimensional smoothing function, which the integral of in the entire plane is 1 , and $\mathrm{x}$ or $y$ is infinite distance converges to 0 . Wavelet function are the two first order derivative's along directions of the $\mathrm{x}$ and $\mathrm{y}$.

$$
\psi^{(1)}(x, y)=\frac{\partial \theta(x, y)}{\partial x}, \psi^{(2)}(x, y)=\frac{\partial \theta(x, y)}{\partial y} 。
$$

So two component for convolution-type wavelet transform in scale for N:

$$
\begin{aligned}
& W T_{N}^{(1)} f(x, y)=f(x, y) * \psi_{N}^{(1)}(x, y) \\
& =N \frac{\partial\left[f(x, y) * \theta_{N}(x, y)\right]}{\partial x} \\
& W T_{N}^{(2)} f(x, y)=f(x, y) * \psi_{N}^{(2)}(x, y) \\
& =N \frac{\partial\left[f(x, y) * \theta_{N}(x, y)\right]}{\partial y}
\end{aligned}
$$

where $*$ is convolution operation, $\mathrm{N}=2$.

$$
\begin{aligned}
& {\left[\begin{array}{ll}
W_{N}{ }^{1} f & (x, y) \\
W_{N}{ }_{N}^{2} f(x, y)
\end{array}\right]=2^{j}\left[\begin{array}{ll}
\frac{\partial}{\partial x}\left(f * \theta_{N}\right)(x, y) \\
\frac{\partial}{\partial y}\left(f * \theta_{N}\right)(x, y)
\end{array}\right] } \\
= & N \nabla\left(f * \theta_{2}\right)(x, y)
\end{aligned}
$$

In the point, vector modulus of :

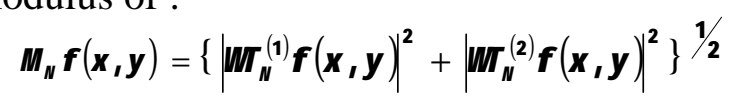

the angle between Gradient vector and horizontal shaft:

$$
A_{N} f(x, y)=\arctan \left(\frac{W_{N}^{(2)}(x, y)}{W_{N}^{(1)_{f}(x, y)}}\right)
$$

The trend of the edge of wood texture image rings is perpendicular to gradient direction vector. Rings edge corresponds to the direction of amplitude angle which is modulus maxima points. According to analyzing maximum points corresponding mode and the angle can get multi-scale edge image. 


\section{Multiscale Texture Analysis and Improved Algorithm}

Algorithm mentioned is for the more common edge detection methods of wavelet modulus maxima. Threshold selection, scale size and edge blending need to further be improved in the edge detection of wood texture.

(1) Threshold Calculation

Because the false edges information are stored in second or third layers of wavelet details in wavelet analysis and each layer threshold should decreased when the larger scale. The author used setting the local stratified threshold. If th is the initial threshold and $n$ is layers, then each layer threshold determined by

$$
t h_{n}=t h_{0} \times\left(1-0.2 \times 2^{n}\right) .
$$

(2) Edge Blending

When each scale of edge information has been detected, edge blending can obtain the complete edge image. The study found that the main factor is wood texture rings. If the traditional adjacent layer edge blending method is used in wood texture. Edge will become blurring and texture feature is not obvious in the image which the interval among ring texture is small. In view of this situation, the author used expansion method which the pixel threshold is three for edge blending.

\section{Fourier Spectrum Analysis Texture Direction}

By means of the Fourier spectrum frequency characteristic, spectrum method describes directivity of two-dimensional model image model which is cycle or approximate cycle. It can reflect the information of the texture direction. If the texture image has strong component toward a direction, high amplitude value of $\mathrm{F}(\mathrm{u}, \mathrm{v})$ will be arranged in a particular direction and the direction and texture direction are perpendicular. If texture direction going is not obvious, $\mathrm{F}(\mathrm{u}, \mathrm{v})$ has not any explicit directions. In figure 3, the spectrum diagram is desultorily and energy cannot be concentrated for the random texture.

\section{Algorithm Principle of Fourier Spectrum}

Two dimensional discrete Fourier transform (2DFT) in image is defined as

$$
F(u, v)=\frac{1}{M N} \sum_{x=0}^{M-1} \sum_{y=0}^{N-1} f(x, y) \exp \{-2 \pi j(u x / M+u y / N)\}
$$

where $=0,1, \ldots, \mathrm{M}-1 ;=0,1, \ldots, \mathrm{N}-1$;

The definition of two-dimensional discrete Fourier transform is complex number, we recorded as :

$$
\begin{gathered}
F(u, v)=R(u, v)+j I(u, v) \\
R(u, v)=\frac{1}{M N} \sum_{x=0}^{M-1} \sum_{y=0}^{N-1} f(x, y) \cos \{2 \pi(u x / M+u y / N)\} \\
I(u, v)=-\frac{1}{M N} \sum_{x=0}^{M-1} \sum_{y=0}^{N-1} f(x, y) \sin \{2 \pi(u x / M+u y / N)\}
\end{gathered}
$$

Where $R(u, v)$ and $I(u, v)$ are $F(u, v)$ real part and imaginary part, Two dimensional discrete Fourier transform spectrum:

The power spectrum:

$$
|F(x, y)|=\left[R^{2}(x, y)+I^{2}(x, y)\right]^{1 / 2}
$$

$$
P(u, v)=\left|F^{2}(x, y)\right|=R^{2}(u, v)+I^{2}(u, v)
$$

Power spectrum represents signal energy of the image in the spatial distribution of frequency domain.

\section{Global Texture Direction Algorithm Based on The Power Spectrum}

Spectrum diagram energy focused on a certain direction of the texture, namely direction of the energy concentration is perpendicular to the texture orientation. Fourier spectrum diagram can reflect orientation of the original texture image. It is possible to calculate the original texture direction from corresponding Fourier spectrum image. It does not require accurate processing in texture synthesis 
field, as long as meeting the required for the human eye vision. A texture image for square image $f_{M N}(i, j)$, two-dimensional Fourier transform to get power spectrum $\mathrm{P}_{\mathrm{M}}(\mathrm{u}, \mathrm{v})$, where $\mathrm{u}, \mathrm{v}=0,1, \ldots, \mathrm{M}$.

According to the power spectrum, the author puts forward a the power spectrum method based on threshold value to texture direction algorithm. The power spectrum $\mathrm{P}_{\mathrm{M}}(\mathrm{u}, \mathrm{v})$ transforms for polar coordinates the function $_{P}(\theta)=\sum_{i=1}^{n} P\left(r_{i}, \theta\right), \theta=\theta_{1}, \theta_{2}, \ldots, \theta_{n}, \theta_{j+1}-\theta_{j}=\pi / n$. To set up fixed value $\theta$, r sums to get image energy is in 0 to 180 degrees.

Setting the power threshold $P_{t h}$, the continuous variation of the group $P(\theta)$ is greater than the power threshold $P_{t h}$, when $\theta$ changed in the 0 to 180 degrees. $r_{i}=\sum_{k=k_{1}}^{k_{2}} P_{k}(\theta)$ are obtained by accumulating. $\quad\left(\mathrm{P}_{\mathrm{k}}(\theta)>\mathrm{P}_{\mathrm{th}}, 0<\mathrm{k}_{1}<\mathrm{k}_{2}<180\right.$, group number ); Between $\mathrm{k}_{1}$ and $\mathrm{k}_{2}$, the characterization of the angle $\theta_{i}$ is $P(\theta)$ corresponding maximum angle. That satisfies conditi $\mathrm{P}_{\mathrm{k}}\left(\theta_{\mathrm{i}}\right)=\max \left(\mathrm{P}_{\mathrm{k} 1}(\theta), \mathrm{P}_{\mathrm{k} 1+1}(\theta), \ldots, \mathrm{P}_{\mathrm{k} 2}(\theta)\right)$. According $r_{i}$ and $\theta_{i}$ can get the characterization of the texture vector direction $\overrightarrow{r_{i}}$. For each texture vector summation can get $\overrightarrow{r_{f}}=\sum_{i=1}^{n} \overrightarrow{r_{i}}$ global vector. We approximate admit that the global vector angle is corresponding texture principal direction.

\section{Texture Principal Direction Algorithm}

The combination of these algorithms, the process which given texture image extraction global grain principal direction algorithm is as follows:

(1) Input a texture image, wavelet maxima edge detection

(2) Expansion maximum edge with threshold 3

(3) Getting power spectrum image

(4) Getting many groups of texture power vector with power threshold. Groups of vector modulus are superposition of power. In the group's angle range, angle is corresponding to the maximum power angle. Finally, the power vector summation gets global power vector, and the angle is texture principal direction.

\section{Analyzing Simulated Results}

Figure 1 shows analysis image of wavelet maxima edge at different scales. Wood texture edge is clear without losing any main visual information on a scale of twenty. It has more details and noise obviously on a scale of ten. Edge information lost obviously on a scale of forty,and Dense ring will lost edge information on the scale, so the author used scale of twenty for wavelet analysis.

The main texture for 85 degrees ring texture and 70 degree nodules texture can directly been seen from figure $2 \mathrm{a}$. There are more Noise points and the ring edge is implicit in figure 4b. After expansion on the threshold of three pixel, it can be seen visually identify the edge blending. Analysis data of image power spectrum in figure 3(using the image power on threshold of 80000) shows that the main texture direction is 85 degrees, the same as visual judgment.

Three main direction of texture visually identify three degrees are 70,85,140 in figure 4a. There are more noise points and can't distinguish ring edges in figure 4b. After distension treatment, ring edges can be obviously resolution. The 85 degree angle is implicit in Image power spectrum showed in figure 5 . In figure 4, power spectrum is amplified greatly, after edge expansion in figure 4c. Three directions can be distinguished; principal direction for 142 degrees is completely consistent with visual judgment. 

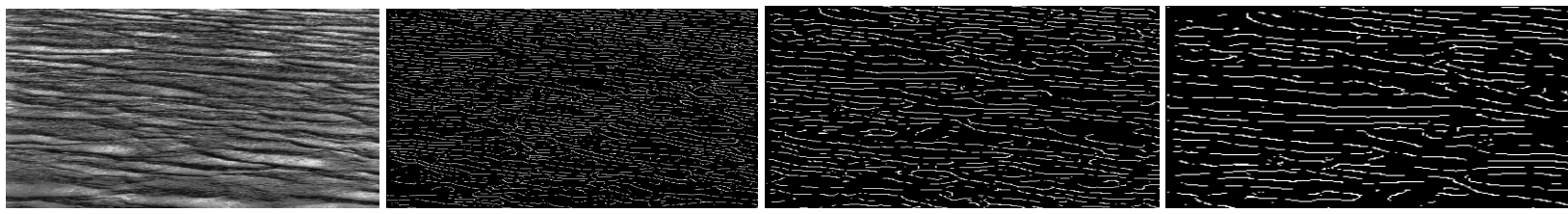

Figure1 D37 Image edge detection based on wavelet modulus maxima

(A) the original image (B) $\mathrm{N}=10$;

(C) $\mathrm{N}=20$;

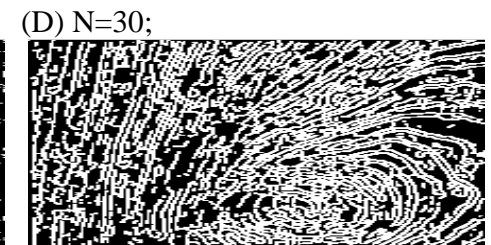

Figure2 D69 Image edge detection based on wavelet modulus maxima

$\begin{array}{lll}\text { (A) the original image } & \text { (B) the image before expansion } & \text { (C) the image after expansion }\end{array}$
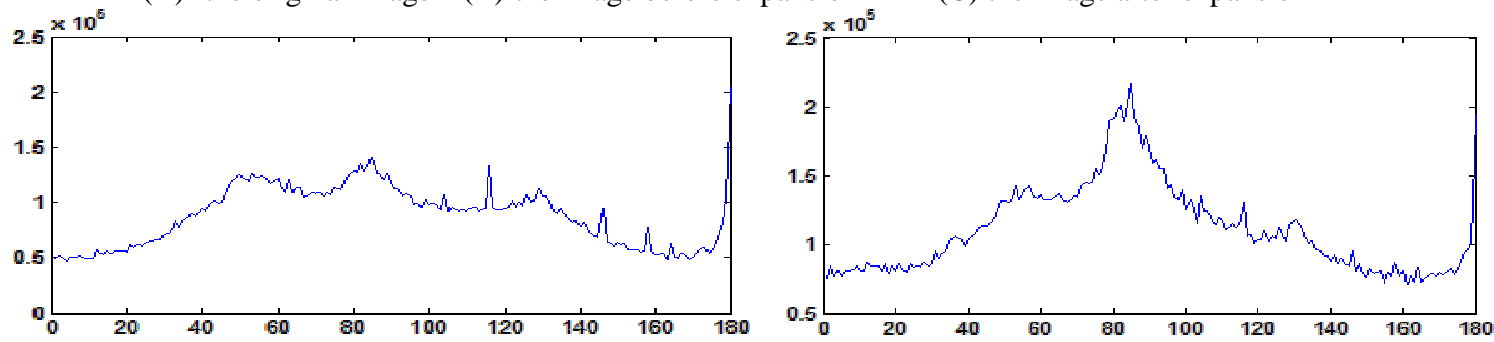

Figure 3 D69 the image power spectrum

(A) image power spectrum before the expansion

(B) image power spectrum after the expansion
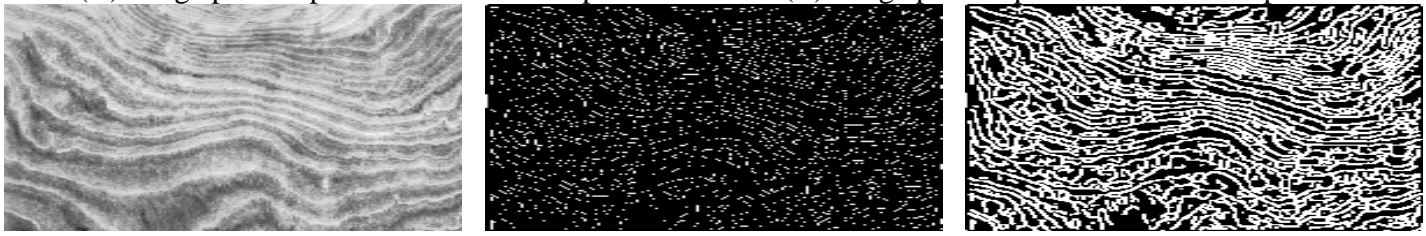

Figure 4 D71 mage edge detection based on wavelet modulus maxima

(A) the original image

(B)the image before expansion

(C) the image after expansion
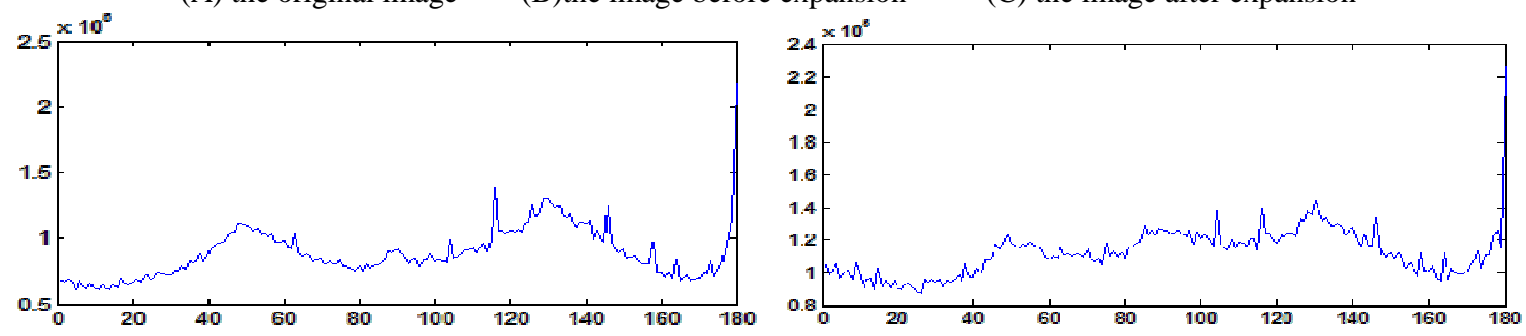

Figure 5 D71 the image power spectrum

\section{Conclusion}

The proposed algorithm can effectively extract edge of wood texture, and for reasonable image fusion and extracting the global power vector in the power spectrum. Algorithm makes full use of the image texture feature. Not only maintain a good edge resolution, but take account of the image keeping characteristics of the whole texture direction.

The test result shows that the paper proposed global texture direction metric algorithm which can satisfy the higher accuracy requirement of man-machine texture recognition model.

\section{References}

[1] Deans S.R. 1983. The Radon transform and some of its application [M]. John Wiley \& Sons, INC.

[2] Guo H.W., Wang, Y., Yang, F.F., Liang, D.N. 2003. Linear chirp signals detec-tion by Wavelet-Radon transform [J]. Journal of National University of Defense Technology, 25(1): 91-94.

(in Chinese) 
[3] Rey M.T., Tunaley J.K.E.,Folinsbee J.T., et al . 1990. Application of Radon transform techniques to wake detection in Seas at a SAR images [J]. IEEE Trans. Geosci. Remote Sensing, 28(4): 553-560.

[4] Wang, J.P. 1999. Some properties of Radon operator [J]. Acta Scientiarum Naturalium Universitatis NeiMongol, 30(6): 675-676. (in Chinese)

[5] Wang, S.Q, Jin, Y.Q. 2001. Ship wake detection in SAR images based on Radon transformation and morphologic image processing [J]. Journal of Remote Sensing, 5 (4): 289-294. (in Chinese) 